\title{
Religionswissenschaftliche Operationalisierungen diskurstheoretischer Elemente im deutschsprachigen Raum
}

Christian Funke and Lisa Züfle

\section{(2) OpenEdition \\ 10 Journals}

Electronic version

URL: http://journals.openedition.org/zjr/714

DOI: $10.4000 /$ zjr.714

ISSN: 1862-5886

Publisher

Deutsche Vereinigung für Religionswissenschaft

\section{Electronic reference}

Christian Funke und Lisa Züfle, « Religionswissenschaftliche Operationalisierungen diskurstheoretischer Elemente im deutschsprachigen Raum », Zeitschrift für junge

Religionswissenschaft [Online], 4 | 2009, Online erschienen am: 31 Dezember 2009, abgerufen am 02 Mai 2019. URL : http://journals.openedition.org/zjr/714 ; DOI : 10.4000/zjr.714

This text was automatically generated on 2 mai 2019

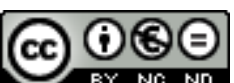

Dieses Werk ist lizenziert unter einer Creative Commons Namensnennung - Nicht-kommerziell - Keine Bearbeitung 3.0 Deutschland Lizenz. 


\title{
Religionswissenschaftliche Operationalisierungen diskurstheoretischer Elemente im deutschsprachigen Raum
}

\author{
Christian Funke and Lisa Züfle
}

\section{Einleitung}

1 Als Modewort, als Mittel der sophistication und als Chiffre scheint »der Diskurs« ein Eigenleben zu führen, das häufig zur Legitimation und Abgrenzung wissenschaftlicher Arbeiten von vermeintlich ideologischen oder sonst untergeordneten Ansätzen dient. Häufig erscheint sein Gebrauch beliebig und »Diskurs« mutet meist als bloßes Füllwort an. Der Diskurs ist zu einer postmodernen Allzweckwaffe mutiert.

Dies hängt wohl auch mit den Schwierigkeiten zusammen, die sich im Laufe der Genese und Verwendung des Diskursbegriffs ergeben haben. Im Folgenden soll daher zunächst ein konziser Rekurs auf die Begriffsgeschichte genommen werden, um anschließend auf seine Verwendung im deutschsprachigen religionswissenschaftlichen Raum überzuleiten. Hierbei stellt der vorliegende Artikel insbesondere die Studien Kippenbergs (1983) und Stuckrads (2003) vor, deren Autoren sich als prominente Exponenten sowohl der deutschsprachigen Religionswissenschaft als auch der religionswissenschaftlich applizierten Diskurstheorie auszeichnen und weite Rezeption erfahren haben. Dabei soll immer auch die Rückbindung an den Foucaultschen Diskursbegriff im Auge behalten werden.

3 Im weiteren Verlauf sollen die Möglichkeiten, die der Diskursbegriff und seine theoretischen Implikationen, die er für die Religionswissenschaft mit sich bringt, theoretisch erörtert und exemplifiziert werden. 


\section{Entwicklung des Diskursbegriffs}

\section{Begriffsgeschichte}

"Diskurs« leitet sich ab vom lateinischen discursus mit den Grundbedeutungen »das Auseinanderlaufen« oder "das Hin- und Herlaufen«. In klassischer Zeit ist eine Verwendung im Sinne von Rede oder Gespräch nicht belegt. In der scholastischen Logik und Erkenntnistheorie hingegen konzeptionalisiert "Diskurs" das Denken als das "Hinund Herlaufen« des Verstandes und bezeichnet als terminus technicus das menschliche Verstandes- und Vernunftvermögen. Ebenfalls überliefert ist die mittellateinische Verwendung von discursus mit »Argument«. Zum Gegenstand von Reflexionen über Sprache, im Sinne mündlicher Rede und schriftlicher Abhandlung, wird der Begriff erstmals in der Philosophie der italienischen Renaissance (Schalk 1997, 81-82).

Ab dem zwanzigsten Jahrhundert umfasst die Bedeutung von »Diskurs« nun- mehr auch die gesellschaftliche und soziale Dimension von Sprache und gewinnt dadurch ein Vielfaches an Komplexität. Durch die unterschiedlichen Ansätze, die sich inhaltlich einerseits voneinander abgrenzen und andererseits schwer voneinander abgrenzbar sind, ist daher die kontextuelle Verortung von »Diskurs« notwenig. Schalk konstatiert:

"Für den Diskursbegriff selbst gilt, was innerhalb der diskursanalytischen Verfahren das entscheidende Kriterium der Sprach-oder Textanalysen ist: Der Kontext entscheidet über die Begriffsbedeutung." (Schalk 1997, 104)

Der Diskursbegriff, der in der Religionswissenschaft wohl am häufigsten Verwendung findet, verdankt sich vor allem strukturalistischen und poststrukturalistischen Ansätzen. Hier nehmen die Sprachtheorie Ferdinand de Sausures (1857-1913) und die diskursanalytischen Arbeiten Michel Foucaults (1926-1984) die dominierende Rolle wahr (Keller 2007, 14-15). ${ }^{1}$

7 Innerhalb des Diskursmodells wird zudem zwischen Theorie und Analyse des Diskurses unterschieden. Unter Diskurstheorie wird meist das Entwickeln allgemein theoretischer Grundlagenperspektiven und deren Anwendung »auf die sprachförmige Konstituiertheit der Sinnhaftigkeit von Welt« (Keller 2007, 8) verstanden. Diskursanalysen konzentrieren sich dabei auf die empirische Untersuchung von Diskursen. Diskursanalyse ist keine spezifische Methode, sondern regelrecht eine Forschungsperspektive mit Diskurs als Gegenstand. Keller bemerkt dazu:

»Diskurstheorien und Diskursanalysen verstehen sich heute meist als qualita- tive,

hermeneutische bzw. interpretative Perspektiven oder werden diesen in

Methodenüberblicken zugeordnet.« (Keller 2007, 8)

\section{Michel Foucault und der Diskurs}

8 Der Diskursbegriff ist - wie erwähnt (und wie jeder andere Begriff) - in starkem Maße durch seinen Kontext, aber auch dadurch definiert, wozu er in Opposition gebracht wird. ${ }^{2}$ Wenn Foucault voraussetzt, dass in jeder Gesellschaft die Produktion des Diskurses zugleich kontrolliert, selektiert, organisiert und kanalisiert wird, um seine Unberechenbarkeit zu bannen, und er behauptet, dass nicht alles jederzeit und von jedem sagbar ist und dazu Prozeduren der Ausschließung, die im Verbot kulminieren können, existieren, so hat er offensichtlich nicht die Regeln rein linguistischer Diskurse im Sinn. Foucault legt seinen analytischen Fokus nicht darauf, den Diskurs als eine Gruppe von 
Zeichen oder als ein Textsegment $\mathrm{zu}$ betrachten, sondern Diskurse in ihrer Wechselwirkung mit Macht, Wissen und Wahrheit zu analysieren (Foucault 2007 [1971], 17-20).

Sein Diskurskonzept expliziert Foucault vor allem in der Archäologie des Wissens (1981 [1969]). Dort bezeichnet er Diskurse als »die Gesamtheit der Aussagen, die das Subjekt der Diskurse (ihr eigenes Subjekt) zum ১Objekt` gewählt haben« (Foucault 1981, 46). Diskurse sind dabei nicht bloße sets von Aussagen, die sich um ein bestimmtes Thema gruppieren und sich als eine Konstellation von institutionell-, zeit- und ortsbedingten Aussagen darstellen, sondern werden von Foucault als selbstständige Einheiten verstanden, die diskursspezifische Effekte produzieren. Die Regeln des Diskurses sind dabei nur bedingt von externen, z. B. sozio-ökonomischen, kulturellen etc., Faktoren abhängig. Sie sind ein Spezifikum und Charakteristikum des Diskurses selbst. Der Diskurs umfasst dabei auch nichtsprachliche Kategorien sowie performative Aspekte (Institutionen und Ereignisse).

Dabei zeitigen Diskurse Wirkung, weil sie in einem engen Verhältnis zu Wahrheit, Macht und Wissen stehen. Für Foucault ist »Wahrheit« dabei keine transzendentale oder ontologische Kategorie. Stattdessen betont er, dass sich jede Gesellschaft eigener Wahrheitsregime bedient, indem sie bestimmte Diskurse annimmt und andere ablehnt. »Wahrheit« wird demgemäß "produziert« und die Prämissen ihrer Erzeugung können untersucht werden (Mills 2007, 19). Auch Norman Fairclough rekurriert in Discourse and So cial Change (1992) auf dieses Modell und exemplifiziert es anhand des Konflikts zwischen Schul- und Alternativmedizin: Indem sich die Schulmedizin spezifischer Diskurse der Wissenschaftlichkeit bedient, versichert sie sich gegenüber der alternativen Medizin, die sie gleichsam als unwissenschaftlich und amateurhaft deklassiert. Dabei produziert der schulmedizinische Diskurs auch eine Demarkationslinie der Selbstversicherung und ermöglicht eine Schablone, anhand deren sich Fremdaffirmation aufbauen lässt.

11 Foucaults Auffassung vom Verhältnis zwischen Diskurs und Wirklichkeit ist ferner dadurch geprägt, dass »Wirklichkeit» nicht definiert wird, da es die diskursiven Strukturen sind, die die Wahrnehmung der Wirklichkeit maßgeblich determinieren. Dabei wird Wirklichkeit als solche nicht in Frage gestellt, jedoch, dass sie sich »außerhalb jeder diskursiven Bedingung ihres Auftretens konstituieren« kann (Mills 2007, 52). Ob ein Blitzschlag als naturwissenschaftliches Phänomen oder als göttliche Handlung aufgefasst wird, stellt seine Wirklichkeit nicht in Frage, doch ist diese Wirklichkeit nachgeordnet, sobald sie konzeptionalisiert wird. Die Wirklichkeit ist somit zwar Vorraussetzung für ihre eigene Strukturierung, gleichsam aber ein Produkt des Diskurses.

Zusammengefasst bedeutet dies: i.) Diskurse sind das in der Sprache erscheinende epochaltypische Verständnis von Wirklichkeit und ii.) die Strukturen des Diskurses definieren, was jeweils sagbar ist und damit wissbar ist.

Letztlich ist Foucault, der wohl auch in der religionswissenschaftlichen Diskursrezeption die prominenteste Stellung einnimmt, bei der theoretischen Ausdifferenzierung und Vereinheitlichung der Diskurstheorie in vielerlei Hinsicht vage geblieben. Dies scheint er nicht intendiert zu haben: In einer Metapher beschrieb er seine Bücher als »kleine Werkzeugkisten«, als "Schraubenzieher oder Schrauben- schlüssel zum Kurzschließen, Diskreditieren oder Zerstören der Machtsysteme« (zitiert nach Mills 2007, 18). ${ }^{3}$ In Foucaults Werkzeugkiste finden wir noch keine religionswissenschaftliche Methode, dafür aber methodologische Ansätze, die von Religionswissenschaftlern aufgegriffen wurden. 


\section{Diskurstheorie und Religionswissenschaft}

\section{Die Diskurstheorie als Werkzeug religionswissenschaftlicher Analyse}

In der deutschsprachigen Religionswissenschaft lassen sich im Wesentlichen zwei Vertreter einer Diskurstheorie finden, Hans G. Kippenberg ${ }^{4}$ und Kocku von Stuckrad ${ }^{5}$. Beide Wissenschaftler tragen rezent zu einer lebhaften Debatte über die Anwendung eines Diskursmodells bei (z. B. Luchesi \& Stuckrad 2004). Zudem wird eine in der deutschsprachigen Religionswissenschaft häufig rezipierte diskurs- analytische Feldstudie des Ethnologen Werner Schiffauer ${ }^{6}$ vorgestellt.

Kippenberg setzt sich erstmals 1983 mit dem Thema auseinander und entwickelt in seinem Aufsatz "Diskursive Religionswissenschaft. Gedanken $\mathrm{zu}$ einer Religionswissenschaft, die weder auf einer allgemein gültigen Definition von Religion noch auf einer Überlegenheit von Wissenschaft basiert« eine Arbeitsdefinition, in der er ein Diskursmodell einem erkenntnistheoretischen Modell entgegen- setzt. Den Ausgangspunkt bildet hierbei die Theorie der Sprechakte, die eine essentialistische Trennung von Handlung und Vorstellung überwindet, indem sie Äußerungen als Handlungen versteht. In der Religionsgeschichte wurden Vorstellungen (beliefs) laut Kippenberg bisher nur in ihrer Rolle als kosmologische Erklärungen und nicht in ihrer Rolle als Rede verstanden. Kippenberg kritisiert diese einseitige Betrachtung. Indem er das erkenntnistheoretische Modell zugunsten der Sprechakttheorie verlässt, treten an die Stelle unmittelbarer Evidenz Geltungsansprüche im Diskurs. So werden durch die Betrachtung religiöser Aussagen in ihrer Rolle als Rede sozialgeschichtliche Vorraussetzungen freigelegt.

Der Verdienst einer Diskurstheorie in der Religionswissenschaft ergibt sich für Kippenberg zudem im Überwinden eines Methodenproblems. In der Anwendung des Diskursmodells findet eine Reflexion über den "unerschütterlichen Beobachterstandpunkt « statt, indem im Prozess des Begreifens Teilnehmer- und Beobachterperspektive notwendigerweise im Gespräch ausgetauscht werden. Kippenberg tritt hier zwar für eine diskursive Religionswissenschaft ein, bleibt in seiner theoretischen Differenzierung jedoch vage.

17 Auch Stuckrad plädiert für ein Diskursmodell in der Religionswissenschaft, betont dabei in seinem Aufsatz »Discursive Study of Religion: From States of the Mind to Communication and Action « jedoch:

»My presentation provides just a very sketchy picture of future research programs and is not intended as a coherent theory.« (Stuckrad 2003, 262) $\mathrm{Er}$ wendet sich gegen die Herangehensweise, eine Definition von Religion $\mathrm{zu}$ normatisieren. Stattdessen tritt er für eine Metatheorie bzw. eine integrative Theorie der Religionswissenschaft in Gestalt einer Diskurstheorie ein.

In ihrem Versuch, sich zu definieren, war die Religionswissenschaft in der Vergangenheit mit drei wesentlichen Problemen konfrontiert, welche Stuckrad zusammenfasst als die "Krise der Repräsentation «" , die Kritik an der Objektivität des Beobachterstandpunktes, die zur Einführung der "teilnehmenden Beobachtung" führt und das "Dilemma von Essentialismus und Relativismus« (Stuckrad 2003, 255). 
21 In Auseinandersetzung mit den angesprochenen Themen haben sich für die Religionswissenschaft dabei wesentliche Einsichten herauskristallisiert. Zum einen das Bewusstsein über den Ethnozentrismus, die Normativität und die Grenzen wissenschaftlicher Erkenntnis. Damit einher geht die Reflexion über die ethnische Subjektivität, welche den impliziten universalen Gültigkeitsanspruch von Tradition aufzudecken vermag. Und schließlich die Feststellung darüber, dass universelle Gültigkeiten immer auf Generalisierungen von lokal und zeitlich begrenzten Phänomenen basieren. Eine radikale Reflexion dieser Erkenntnisse bringt Stuckrad zu der Frage nach der Entstehung von Wissen (Stuckrad 2003, 261).

Was für die Religionswissenschaft somit eine reale Basis der Untersuchung dar- stellt, ist der Ort, an dem Religion tatsächlich sichtbar ausgedrückt wird, ergo auf der Ebene von Kommunikation und Handlung. Stuckrad bezieht sich hier, anders als Kippenberg, auf die Theoriebildungen von Roland Barthes, Michel Foucault und Pierre Bourdieu und somit einen Diskursbegriff im kulturwissenschaftlichen Sinne:

»From this perspective, discourse conceptualizes representations of social positions that are negotiated among groups in a complex process of identity formation and demarcation.« (Stuckrad 2003, 266)

Dabei werden Kontinuitäten, Aneignungen, Bedeutungsverschiebungen und Machtbeziehungen sichtbar, welche die Akteure in einem Diskursfeld unter Anwendung bestimmter Regeln und Ressourcen aushandeln und ausdrücken.

Religiöse Traditionen werden so in ihrer Mehrdimensionalität als dynamische Netzwerke, bestehend aus Identitäten und Bedeutungen sichtbar und zeigen sich vor diesem Hintergrund als mächtige Bestandteile des öffentlichen Diskurses.

»Religions are powerful because they serve as instruments in the communica- tive

formation of identity and provide people with a concrete script of action.« (Stuckrad 2003, 269)

Statt Religionen also als (nicht nachweisbare) Glaubenssysteme zu definieren, begreift von Stuckrad sie als Kommunikations- und Handlungssysteme. Dazu sollen öffentlich kommunizierte Konstruktionen statt innere Seelenzustände und Spekulationen über das Transzendente Gegenstand sein und analysiert werden. Die Stärke der Anwendung des Diskursmodells liegt also darin, dass Diskursfelder die gleichzeitige Wahrnehmung von diesen kulturellen Arenen als auch die rekursive Teilnahme der Wissenschaftler, die selbst Akteure darin sind, ermöglichen (Stuckrad 2003, 255).

Ein in der Religionswissenschaft oftmals herangezogenes Beispiel für die praktische Anwendung einer Diskursfeldanalyse stellt die Forschung des Ethnologen Werner Schiffauer dar. Dieser beschreibt in seiner ethnologischen Studie Die Gottesmänner. Türkisc he Islamisten in Deutschland (Schiffauer 2000) die Geschichte einer radikalen Gruppe türkischer Muslime in Köln, der Kaplan-Gemeinde. Dabei spricht er sich in seiner Betrachtung vehement gegen einen überlegenen Beobachterstandort und damit gegen die unreflektierte Normativität der Wissenschaften aus. Stattdessen verfolgt er einen konstruktivistischen und relativistischen Ansatz, indem er den Gegenstand seiner Untersuchung als Diskursfeld entwirft. Anhand der Analyse des Diskursfeldes dekonstruiert der Autor den Fundamenta- lismusbegriff und die essentialistische Darstellung religiöser Gruppierungen. Er zeigt eindrücklich die Dynamik des religiösen Denkens, indem er die von ihm untersuchte Gemeinschaft "als ein offenes Netzwerk von sich immer neu gruppierenden Gläubigen, in dem über Deutungen und Bedeutungen gestritten wird«, dar- stellt (Schiffauer 2000, 320). Durch diese Herangehensweise wird 
außerdem deut- lich, dass sich die Positionen der einzelnen Akteure nur durch die Analyse dieses dynamischen Zusammenspiels im Diskursfeld verstehen lassen.

Der Diskursbegriff wird für die Religionswissenschaft demnach in zweierlei Hinsicht relevant: Erstens kann die Religionswissenschaft selbst als Diskursfeld analysiert werden und zweitens kann der Gegenstandsbereich der Religionswissenschaft diskursiv gefasst werden.

\section{Religionswissenschaft als Diskursfeld}

Foucaults Feststellung, dass sich die eigenen Positionen nicht vollständig außerhalb der von ihm analysierten Ideen und Praktiken bewegen, impliziert, dass alle (wissenschaftlichen) Aussagen durch die diskursiv strukturierten und institutionalisierten Zwänge und Machtbeziehungen geformt und determiniert sind, deren Teil sie sind. Für eine Übertragung des Elements wissenschaftlicher Kritik auf diese Konstellation bedeutet dies, dass jede Aussage, die sich gegen eine Form der Unterdrückung richtet, gleichzeitig Teil der Unterdrückung ist. Die Kritik, z. B. an einem Wirtschaftssystem, ist durch die diskursiven Strukturen, die innerhalb des jeweiligen Systems verortet sind, mitdeterminiert. ${ }^{8}$ Damit kannibalisiert Foucault auch das Konzept eines »autonomen Subjekts «. ${ }^{9}$

Gleichzeitig bedeutet diese Erkenntnis, dass diskurstheoretisch grundierte Analysen anderer Kulturen, da sie innerhalb der diskursiven Umwelt des Analytikers stattfinden, $\mathrm{zu}$ einer Restrukturierung und Reorganisierung, einer Über-Setzung nicht nur des Fremden, sondern auch des Eigenen führen. Der Botaniker, der die Flora Sumatras dem Klassifikationsschema von Linné unterwirft, sie gleichsam »ent-deckt« und benennt und dadurch erst - mit deutlichen Parallelen zum jüdisch-christlichen Schöpfungsakt existent macht, zeitigt Sekundäreffekte, die diskurstheoretisch durchleuchtet werden können. Nicht die Klassifikation stünde im Vordergrund der Betrachtung, sondern die diskursiven Strukturen, die sie her- vorbringen, und die Wechselwirkung mit den diskursiven Strukturen, auf die sie trifft. Sag-, und damit wissbar, ist nur das Eigene, das Fremde wird mittels bekannter Schablonen (neu-)gezeichnet, indigene Muster werden ignoriert. Der Vorteil der Diskurstheorie ist hierbei, dass sie - im Gegensatz zu rezeptionsgeschichtlichen Modellen - die Wechselwirkung von Macht und Wissen explizit einbindet und ausdrücklich verlangt.

Diese Verbindung zwischen Religionswissenschaft und ihren Untersuchungsobjekten zeigt sich eindrucksvoll an der Einführung des Begriffes "Religion« und seine Übertragen auf außereuropäische Traditionen. Der Begriff des Hinduismus wird zunächst durch die britische Kolonialmacht im Rahmen der Volkszählung des Jahres 1881 in Indien eingeführt. Zuvor geschah die Selbstzuschreibung der nun als »Hindu« Benannten meist über die Kategorie »Kaste« oder über die Volks- gruppe. Dies wandelt sich zu Beginn des 19. Jahrhunderts aufgrund antikolonialer Motive (Michaels 1998, 28-29). Die Kategorie "Hinduismus" wird nun von den so Bezeichneten übernommen und dient als Projektionsfläche der Selbstaffirmation und -konstruktion, um sich gegen zunehmende kolonialistische Einflussnahme als Einheit zu positionieren, und festigt und beschleunigt sich im Spannungsfeld der beginnenden Unabhängigkeitsbewegung Indiens. ${ }^{10} \mathrm{Am}$ Beispiel der Konstruktion des Hinduismus als Weltreligion wird die direkte Beeinflussung der inneren und äußeren Identität dieser Tradition somit besonders deutlich. Stietencron fasst zusammen: 
»Heute weiß man, ohne dies zugeben zu wollen, daß der Hinduismus nichts ist als eine von der europäischen Wissenschaft gezüchtete Orchidee. Sie ist viel zu schön, um sie auszureißen, aber sie ist eine Retortenpflanze: In der Natur gibt es sie nicht.« (Stietencron 1999, 25-26)

31 Die Religionswissenschaft findet sich also selbst, sowohl als »Konsument « und als »Produzent«, in einem Diskursfeld wieder. In den Toleranzgrenzen ihrer wissenschaftlichen Arbeitsweise, im Fundieren von Theorien, Konstruieren von Geschichte, Normieren politischer und sozialer Intentionen und Aushandeln der Machtpositionen zeigt sie sich in die Strukturen sozialer Auseinandersetzungen eingebunden.

32 Als ein weiteres Beispiel, das geradezu als konstituierend für den religionswissenschaftlichen Diskurs bezeichnet werden kann, ist die Religionsdefinitionsdebatte zu nennen. Sie hat als hart umkämpftes Gebiet zu mehreren Neuausrichtungen und Spaltungen innerhalb der Religionswissenschaft geführt. Insbesondere die von einigen Religionswissenschaftlern als kryptotheologisch verbrämte und zahl- reichen Dekonstruktionen unterworfene »Otto-These « ist nicht zuletzt auch ein potentieller und zumindest impliziter Konfliktpunkt im Spannungsrahmen der universitätspolitischen Sonderstellung der Religionswissenschaft zwischen theologischer und philosophischer Fakultät. Neue Religionsdefinitionen und Modifikationen oder Erklärungen $\mathrm{zu}$ deren Unmöglichkeit sind auch immer mit einer Positio- nierung verbunden, die Auswirkungen auf das Fach zeitigt und diesem letztendlich sogar seine Existenzberechtigung abzusprechen vermag. ${ }^{11}$

Das religionswissenschaftliche Diskursfeld erscheint somit als stark umkämpfter Raum in der Produktion von Wissen über Religion und Religionen. Es liegt in der Verantwortung des Faches hier selbstreflexiv und kritisch zu sein, was dazu führen kann, dass sich durch die Betrachtung von Religionswissenschaft als Diskursfeld neue Perspektiven eröffnen: Erstens steht ein Mittel zur disziplingeschichtlichen Aufarbeitung bereit, das es auch ermöglicht, die Aushandlungsprozesse der eigenen und zeitgenössischen wissenschaftlichen Identität $\mathrm{zu}$ reflektieren, und zweitens ermöglicht es die Diskurstheorie, die Wechselwirkungen des eigenen Sprechens/Handelns auf die untersuchten Gegenstände sichtbar zu machen.

\section{Zusammenschau}

Die Diskurstheorie ist trotz aller Probleme als heuristische Kategorie nützlich und auch in der Religionswissenschaft gut operationalisierbar. Im Gegensatz zu rezeptionsgeschichtlichen Ansätzen blendet sie die jeweils gültigen Machtbeziehungen nicht aus. Sie sensibilisiert und erweitert die Wissenschaftskritik, die im Falle der Religionswissenschaft allzu oft in der Nähe eines schwammigen Eurozentrismusbegriffs lauerte und nach unbedarften Opfern Ausschau hielt. ${ }^{12}$

Ein gemeinsames Merkmal der Ansätze von Kippenberg und Stuckrad besteht in einem Plädoyer für ein integratives metatheoretisches Diskursmodell (Stuckrad 2003, 263) und dessen Implementierung in der Religionswissenschaft. In einem solchen werden Religionsgemeinschaften als Diskursgemeinschaften beschrieben und analysiert. Damit hoffen die Autoren, den Problemen einer Religionsdefinition zu entgehen. Kippenberg und Stuckrad tun dies, indem sie sich für die Aufgabe einer Religionsdefinition aussprechen. Sie wenden sich gegen die von ihnen konstatierten Positionen des 
Ethnozentrismus, Essentialismus und die Überlegenheit des wissenschaftlichen Beobachterstandpunkts.

Auffällig sind dabei die augenscheinlichen Probleme, die der Diskursbegriff bereitet. Während das Problem der Religionsdefinition scheinbar behoben wird, verlagert es sich tatsächlich nur auf das Problem einer Diskursdefinition. Indem diese Definitionsschwierigkeiten aber ausgetauscht wurden, scheinen sie sich nur verschärft $\mathrm{zu}$ haben. Die sich als interdisziplinär arbeitendes Fach verstehende Religionswissenschaft sieht sich plötzlich der Notwendigkeit ausgesetzt, einen Dis- kursbegriff zu etablieren, der dem pluralen Umfeld ihres Gegenstandes gerecht wird - ein Phänomen, das bereits die Religionsdefinitionsdebatte zu genüge erfahren hat. Dabei ist jedoch fraglich, ob als Konsequenz der Aufgabe des Religionsbegriffs, der den Verlust des disziplinären Rahmens bedeuten würde, noch von ihrem Gegenstand gesprochen werden kann.

Die Diskurstheorie kann den Religionsbegriff nicht ersetzen. Um das spezifisch Religiöse der Diskurse analysieren zu können, muss es zunächst theoriefähig definiert werden. Umgekehrt ist "Religion" jedoch oft eine unzureichende Kategorie, um religiöse Aushandlungsprozesse und Beziehungen zu beschreiben. Eine mögliche Aufgabe einer religionswissenschaftlichen Diskursanalyse ist demnach die Extrapolation spezifisch religiöser Elemente in Diskursen - auch solcher, die sich nicht dezidiert als religiös zu erkennen geben.

Zudem ergibt sich die Konsequenz, dass die multidisziplinär arbeitende Religionswissenschaft weder zur Herleitung einer einheitlichen Diskurstheorie in der Lage ist, noch einer generellen Theorie bedarf. Die von Foucault intendierte und $\mathrm{zu}$ heuristischen Zwecken geeignete Instrumentalisierung seiner Werkzeugkiste aber eignet sich für religionswissenschaftliche Analysen und führt $\mathrm{zu}$ einer Erweiterung religionswissenschaftlicher Perspektiven.

\section{BIBLIOGRAPHY}

BERGUNDER, Michael (2006). »Die Bhagavadgita im 19. Jahrhundert. Hinduismus, Esoterik und Kolonialismus«. In: Michael BERGUNDER (Hg.): Westliche Formen des Hinduismus in Deutschland. Eine Übersicht. Halle: Franckesche Stiftungen zu Halle, 187-216.

BROWN, Gillian und George YULE (1983). Discourse Analysis. Cambridge: Cambridge University Press.

FAIRCLOUGH, Norman (1989). Language and Power. London: Longman.

FAIRCLOUGH, Norman (1992). Discourse and Social Change. London: Polity.

FITZGERALD, Timothy (2000). The Ideology of Religious Studies. New York, Oxford: Oxford University Press.

FOUCAULT, Michel (1981 [1969]). Archäologie des Wissens [L'archéologie du savoir]. Frankfurt a. M.: Suhrkamp. 
FOUCAULT, Michel (2001 [1975]). »Des supplices aux cellules« [Interview mit R.-P. Droit, in: Le Monde, Nr. 9363, 12. Februar 1975, S. 16]. In: Michel FOUCAULT (herausgegeben von Daniel DEFERT, Fraçois EWALD und Jaque LAGRANGE) (22001[1994)]: Dits et écrits: 1954-1988, Band 1 (1954-1975). Paris: Gallimard.

FOUCAULT, Michel ( ${ }^{10} 2007$ [1971]). Die Ordnung des Diskurses [L'ordre du discours]. Frankfurt a. M.: Fischer.

HABERMAS, Jürgen (1990 [1962]). Strukturwandel der Öffentlichkeit. Untersuchungen zu einer Kategorie der bürgerlichen Gesellschaft. Frankfurt a. M.: Suhrkamp.

KELLER, Reiner ( ${ }^{3} 2007$ [2004]). Diskursforschung. Eine Einführung für SozialwissenschaftlerInnen. Wiesbaden: Verlag für Sozialwissenschaften.

KIPPENBERG, Hans G. (1983). »Diskursive Religionswissenschaft: Gedanken zu einer Religionswissenschaft, die weder auf einer allgemein gültigen Definition von Religion noch auf einer Überlegenheit von Wissenschaft basiert«. In: Burkhard GLADIGOW und Hans G. KIPPENBERG (Hg.): Neue Ansätze in der Religionswissenschaft. München: C. H. Beck, 9-28.

KIPPENBERG, Hans G. und Kocku von Stuckrad (Hg.) (2003). Einführung in die Religionswissenschaft. München: C. H. Beck.

KIPPENBERG, Hans G., Kocku von Stuckrad und Jörg Rüpke (Hg.) (2009). Europäische Religionsgeschichte - ein mehrfacher Pluralismus. 2 Bände, Göttingen: Vandenhoeck \& Ruprecht/UTB.

LINCOLN, Bruce (1989). Discourse and the Construction of Society. Comparative Studies of Myth, Ritual, and Classification. New York, Oxford: Oxford University.

LUCHESI, Brigitte und Kocku von STUCKRAD (Hg.) (2004). Religion im kulturellen Diskurs. Festschrift für Hans G. Kippenberg zu seinem 65. Geburtstag. Berlin: De Gruyter.

MICHAELS, Axel (1998). Der Hinduismus. Geschichte und Gegenwart. München: C. H. Beck. MILLS, Sara (2007 [1997]). Der Diskurs. Begriff, Theorie, Praxis [Discourse. The new Critical Idiom]. Tübingen \& Basel: A. Francke.

PÊCHEUX, Michel (1982 [1975]). Language, Semantics and Ideology [Les vérités de la palice. Linguistique, sémantique, philosophie]. Basingstoke: Macmillan.

SCHALK, Helge. (1997/1998). »Diskurs. Zwischen Allerweltswort und philosophischem Begriff.«. In: Archiv für Begriffsgeschichte 40, 56-104.

SCHIFFAUER, Werner (2000). Die Gottesmänner. Türkische Islamisten in Deutschland. Eine Studie zur Herstellung religiöser Evidenz. Frankfurt a.M.: Suhrkamp.

STIETENCRON, Heinrich von ( ${ }^{21} 999$ [1995]). »Hinduistische Perspektiven«. In: Hans KÜNG und Heinrich von STIETENCRON: Hinduismus. Christentum und Weltreligionen. München: Piper Verlag, 25-52.

STUCKRAD, Kocku von (2003). »Discursive Study of Religion: From States of the Mind to Communication and Action«. In: Method an Theory in the Study of Religion 15, 255-271. 


\section{NOTES}

1. Aber auch etwa die Arbeiten von Michel Pêcheux (1975 et al.), Norman Fairclough (1989 et al.) und Jürgen Habermas (1990 et al.) waren und sind für den kulturwissenschaftlichen Umgang mit Diskurs einflussreich.

2. In der Linguistik wird »Diskurs« - oft in Opposition zu »Text« - als längeres Textsegment oder als linguistische Kommunikation/Transaktion zwischen Sprecher und Hörer verstanden, deren Form durch eine soziale Absicht determiniert ist (Mills 2007, 4). Diese Ansätze sind nicht notwendigerweise ideologiekritisch

3. »Tous mes livres [...] sont [...] de petites boîtes à outils. Si les gens veulent bien les ouvrir, se servir de telle phrase, telle idée, telle analyse comme d'un tournevis ou d'un desserre-boulon pour court-circuiter, disqualifier, casser les systèmes de pouvoir, y compris éventuellement ceuxlà mêmes dont mes livres sont issus... eh bien, c'est tant mieux!« (Foucault 2001 [1975], 1588)

4. Hans G. Kippenberg ist Professor für Religionswissenschaft an der Jacobs-Universität Bremen.

5. Kocku von Stuckrad ist Professor für religiewetenschap an der Faculteit Godgeleerdheid en Godsdienstwetenschap der Rijksuniversiteit Groningen.

6. Werner Schiffauer ist Professor für Vergleichende Kultur- und Sozialanthropologie an der Europa-Universität Viadrina in Frankfurt/Oder.

7. Sie entwickelt sich aus der Kritik an der »Abbildtheorie«, die behauptet wissenschaftliche Beschreibung wäre in der Lage ein Abbild von Realität und Natur zu zeichnen.

8. Dabei ist nicht das System ausschlaggebend, sondern der Diskurs, da sich dieser - im Gegensatz zu »Ideologie« - nicht auf eine Basis bezieht, sondern selbst Subjekt ist.

9. In der Archäologie des Wissens untersucht Foucault gerade auch die Genese der Vorstellung des Subjekts in den westlichen Gesellschaften und entwirft seine geschichtliche Entwicklung visàvis genealogischer Parameter der Wissenskonstitution, der Diskurse etc. ohne dabei auf ein Subjekt zu verweisen (Foucault 1981, 59).

10. Dieser Prozess einer Identitätsbildung lässt sich anhand der Bhagavadgitā-Rezeption des 19. und 20. Jahrhunderts exemplarisch beobachten (Bergunder 2006).

11. Als prominentes Beispiel für diese Extremposition kann Timothy Fitzgeralds The Ideology of Religious Studies dienen (vgl. Fitzgerald 2000, 3-32).

12. Etwas überspitzt könnte man behaupten, dass auch eine überdeterminierte und damit letztlich entleerte Diskurschiffre noch die Wirkmächtigkeit eines warnenden Symbols besitzt und damit in letzter Konsequenz und in impliziten Rückbindungen immer noch zu einer Erweiterung des Aufmerksamkeitshorizontes beitragen kann.

\section{ABSTRACTS}

Der vorliegende Artikel diskutiert die Einsatzmöglichkeiten der Diskurstheorie und ihrer Elemente in der Religionswissenschaft. Dazu bespricht er zunächst die Begriffsgeschichte, um anschließend ein Schlaglicht auf die Foucault'sche Konzeptionalisierung zu werfen. In einem nächsten Schritt werden anhand der deutschen Religionswissenschaftler $\mathrm{H}$. Kippenberg und $\mathrm{K}$. von Stuckrad Beispiele für die disziplinspezifische Anwendung einer modifizierten Diskurstheorie und deren Auswirkungen auf die Methodologie erörtert. Dabei wird deutlich, dass 
die Diskurs- theorie auch als ein Mittel verstanden wird, um nach der Notwendigkeit einer Religionsdefinition $\mathrm{zu}$ fragen. Abschließend wird auf die Möglichkeit verwiesen, die Diskurstheorie als Mittel der Disziplinkritik einzusetzen. Das Fazit des Artikels kommt zu der Feststellung, dass sich die Diskurstheorie zu religionswissenschaftlichen Zwecken eignet, letztlich aber im religionswissenschaftlichen Rahmen eine theoriefähige Religionsdefinition nicht ersetzen kann.

The present article addresses the potential of discourse theory and its elements in religious studies. First, the focus is directed at the conceptual history in order to shed light on Foucault's conceptualisation of discourse. Second, we discuss the dis- cipline-specific implementation of a modified discourse theory and its impact on methodology based on the examples provided by the German scholars H. Kippen- berg and K. von Stuckrad. This approach demonstrates that discourse theory can be conceived as a means to question the necessity of a definition of religion. The final section demonstrates the critical potential of discourse theory. In conclusion, discourse theory is a useful lens through which to gain further insight into the aca- demic discipline of religious studies, but ultimately, is not sufficient to replace a definition of religion applicable for further theorising.

\section{AUTHORS}

\section{CHRISTIAN FUNKE}

Christian Funke studiert seit 2003 Religionswissenschaft und Islamwissenschaft an der RuprechtKarls-Universität Heidelberg und magistriert derzeit. Außerdem studierte er an der Bilkent Üniversitesi in Ankara und betrieb Feldforschungen in İstanbul. Kontakt: christian.funke@ori.uni-heidelberg.de

\section{LISA ZÜFLE}

Lisa Züfle studiert seit 2004 Religionswissenschaft und Ethnologie an der Ruprecht-Karls-Universität Heidelberg. Sie absolvierte mehrere akademische und ethnographische Aufenthalte in Indien.

Kontakt: lisa.zuefle@gmx.de 